\title{
Carnets
}

Revue électronique d'études françaises de l'APEF

Deuxième série - 5 | 2015

Imaginaires de guerre et autres conflits

\section{Résurrection : un espace littéraire pour l'imaginaire en temps de guerre}

\section{Laurence Boudart}

\section{OpenEdition}

1 Journals

\section{Édition électronique}

URL : http://journals.openedition.org/carnets/426

DOI : $10.4000 /$ carnets.426

ISSN : 1646-7698

Éditeur

APEF

\section{Référence électronique}

Laurence Boudart, «Résurrection : un espace littéraire pour l'imaginaire en temps de guerre », Carnets [En ligne], Deuxième série - 5 | 2015, mis en ligne le 30 novembre 2015, consulté le 19 avril 2019. URL : http://journals.openedition.org/carnets/426 ; DOI : 10.4000/carnets.426

Ce document a été généré automatiquement le 19 avril 2019.

\section{(c) (i) (8)}

Carnets est mis à disposition selon les termes de la licence Creative Commons - Atribution - Pas d'utilisation commerciale 4.0 International. 


\section{Résurrection : un espace littéraire pour l'imaginaire en temps de guerre}

Laurence Boudart

\section{Introduction}

1 Seule publication littéraire d'avant-garde à voir le jour en Wallonie pendant la Première Guerre mondiale, Résurrection témoigne de l'incertitude et de l'espoir qui dominent cette période complexe. Point de ralliement de l'expressionnisme européen, la revue rassemble des collaborations de langue française et allemande (en traduction), réunies autour de Clément Pansaers, son actif et zélé directeur. Sans s'y limiter, on y décèle çà et là des manifestations d'un modernisme auquel on associera, après-guerre, le dadaïsme, dont Pansaers sera un des auteurs majeurs, mais longtemps oublié1, et dont on connaît la puissance de subversion, en réaction au choc de la guerre.

2 Revue publiée en Belgique occupée, Résurrection symbolise un moment d'engagement pacifiste par l'écriture, un nouveau départ auquel renvoie son titre ${ }^{2}$; en d'autres termes, elle incarne une autre manière de sublimer la guerre par l'imaginaire. À côté de ces intentions louables, il ne faudrait cependant pas négliger le contexte particulier dans lequel naît et évolue la revue, à savoir celui de l'occupation. Aussi ambivalents, voire parfois confus soient-ils, les propos tenus par Pansaers à propos de la Belgique dans les Bulletins politiques de la revue possèdent des similitudes notables avec les projets que nourrissaient les Allemands ${ }^{3}$. Une telle attitude en période de guerre ne peut dès lors qu'éveiller la suspicion. Sans préjuger de ses convictions idéologiques réelles ${ }^{4}$, il y a fort à penser que la revue est apparue pour Pansaers - inconnu jusque là - comme une opportunité d'étayer sa stratégie de légitimation littéraire. Et pour l'autorité allemande, Résurrection a pu servir à la fois comme canal de propagande et comme un alibi de sa politique culturelle . 


\section{Histoire et évolution de la revue} Cahiers mensuels littéraires illustrés, avec une couverture ornée d'un dessin de Guy Boscart, qui n'est autre qu'un des pseudonymes utilisés par Clément Pansaers. Du premier au dernier numéro, l'adresse indiquée pour joindre la « rédaction et administration » reste identique et correspond au domicile personnel de Pansaers à La Hulpe. Or, le lieu d'édition annoncé - Namur - demeure lui aussi inchangé au long des six numéros. Marc Dachy voit dans ce choix géographique le signe d'une "protestation contre le déclin intellectuel de la Belgique francophone » (Dachy, 1972). On peut aussi y lire une manière de se conformer aux plans fédéralistes de l'occupant, qui avait décidé de faire de Namur la capitale de la partie wallonne de la Belgique. Pour Rik Sauwen (1969: 38-39), il s'agit peut-être d'une manifestation de "l'option wallingante » qu'aurait soutenue Pansaers, comme une «prise de conscience (...) capitale » pour la sauvegarde des Wallons en tant qu'entité politique et identitaire.

Initiateur et directeur de cette publication, Clément Pansaers est né en 1895 à Neerwinden, dans le Brabant flamand actuel. Il s'essaie d'abord à l'écriture en néerlandais, au début des années 1910, sous le pseudonyme de Julius Krekel, entre autres avec une pièce d'inspiration futuriste, Een mysterieuse schaduw [Une ombre mystérieuse]. Dès avant la guerre, Pansaers fréquente à Bruxelles la colonie allemande, dont Hubert Roland a bien décrit le fonctionnement et les avatars ${ }^{6}$. Secrétaire de Carl Sternheim (1878-1942) et précepteur de ses enfants de décembre 1916 à la mi-avril 1918 (Roland, 2003 : 180), Pansaers a fréquenté, outre Sternheim, le poète alsacien de langue et nationalité allemande Ernst Stadler (1883-1914) et l'historien de l'art Carl Einstein (1885-1940), qui lui fera découvrir entre autres Chagall et Kokoschka. Installé dans la capitale belge depuis 1912 (il y décède trente ans plus tard), Sternheim est notamment connu pour son cycle théâtral Aus dem bürgerlichen Heldenleben [Vie héroïque de la bourgeoisie], où il s'applique à parodier la grande bourgeoisie dont il est issu. Avec la guerre, les chemins de ces intellectuels se séparent : Carl Sternheim reste en Belgique, Ernst Stadler meurt à l'automne 1914 près d'Ypres et, après avoir été blessé sur le front, Einstein est renvoyé en Belgique où il est désigné, après sa guérison, comme avocat d'office des soldats allemands au Conseil de guerre, qui siège à Namur.

Pendant la guerre, Clément Pansaers continue de fréquenter ses amis allemands restés en Belgique. En novembre 1918, il affiche son soutien à la cause révolutionnaire du Soldatenrat et participe à quelques mouvements insurrectionnels d'inspiration bolchévique à Bruxelles, restés sans suite (Sauwen, 1969 : 47). Et en 1919, la justice belge le poursuit pour sympathie avec l'ennemi. Après l'Armistice, Pansaers rejoint un temps Sternheim à Berlin ${ }^{7}$ puis marque son adhésion au mouvement Dada ${ }^{8}$ de Tristan Tzara qu'il a découvert grâce à son ami allemand. Son recueil Pan pan au cul du nu nègre (1920) lui vaut les félicitations d'André Breton et Louis Aragon. À Paris où il se rend fréquemment, il collabore à la revue Littérature et rencontre Francis Picabia et Philippe Soupault. Installé au printemps 1921 dans la capitale française, il se sépare du groupe dadaïste au bout de quelques jours'. Atteint de la maladie d'Hodgkin, c'est un Pansaers très affaibli qui coordonne le numéro spécial que la revue anversoise d'avant-garde ça ira! consacre au mouvement Dada. Rentré à Bruxelles, il y décède dans un dénuement total le 31 octobre 1922, à l'âge de trente-sept ans. 
7 La sortie du premier numéro de Résurrection en décembre 1917 coïncide avec l'éclatement de la révolution russe. Loin d'être une simple coïncidence, cet événement majeur déterminera aussi la ligne de la revue, notamment celle que reflètent certains aspects des Bulletins politiques que Pansaers y publie chaque mois. À vrai dire, on ne sait pas grand chose concernant la genèse exacte du projet. Évoquée très rapidement dans un bref texte autobiographique dénommé "Après six mois de méditation sur un aveugle mur blanc ", l'édition de Résurrection s'y voit liée à ce qu'il appelle sa naissance de 1916, survenue après un semestre de réflexion, qui lui fit comprendre « que dans la vie n'est intéressante que la fantaisie chevauchant le hasard» (Pansaers, 1972 : 9). La révélation de ce présupposé métaphysique s'accompagne d'une fréquentation de «jeunes de Bruxelles - peintres, poètes » qui « venaient se reposer dans [s]a bien sentimentale "Maison à l'Orée" ». De ce creuset fécond et des interrogations sur le sens de la vie et le rôle de la littérature naîtra Résurrection.

8 Si l'on dispose de peu d'informations sur sa genèse ${ }^{10}$, il en existe moins encore sur sa disparition. Celle-ci n'est ni annoncée ni même évoquée dans le dernier numéro. Seul indice des menaces qui pèsent sur sa survie, une défection des contributeurs rapidement mentionnée en fin de volume: "Une demi-douzaine de jeunes talents, en des missives enthousiastes - hier - se joignaient à nous. Aujourd'hui, l'un après l'autre, nous écrivent à peu près la même chose : 'une question de relations avec un vilain bourgeois m'oblige à me cacher derrière l'anonymat' ou 'de remettre ma collaboration à une date ultérieure'. » (Pansaers, VI, 1918 : 234) ${ }^{11}$ Pour Michel de Ghelderode, auteur d'une «Introduction aux œuvres complètes de Clément Pansaers », la fin de la revue pourrait aussi être imputable à la disparition de «subsides et de convictions » (Roland, 2003 : 183). Il faut savoir qu'elle avait bénéficié de la bienveillance du service de la censure de la Politische Abteilung, dont le directeur Von der Lancken souligne l'engagement en faveur d'une "séparation politique de la Flandre et la Wallonie, par exemple sous la forme d'une transformation de la Belgique en un État fédéral, consistant en deux États indépendants $\aleph^{12}$, ce qui correspond aux desseins de l'occupant. On peut aussi conjecturer que les prises de position de plus en plus radicales exprimées par Pansaers en faveur d'une internationale communiste ont fini par indisposer les autorités allemandes. Autre hypothèse: en juin 1918, date du dernier numéro, la victoire alliée est pratiquement acquise et, si l'on accepte l'idée que la revue ait pu servir les intérêts allemands, elle ne leur est plus d'aucune utilité.

\section{À propos du contenu ${ }^{13}$}

9 Le premier numéro ne comporte aucun texte programmatique, ce qui semble avoir motivé quelques commentaires, que Pansaers relaie dans le troisième numéro : « Après la lecture des deux premiers cahiers, certains nous demandent le programme de Résurrection . Je réponds, voyez nos actes: probité, équité, franchise.» Ensuite, après avoir cité Flaubert et sa revendication d'un humanisme par-dessus les races et les nations, Pansaers crie son désir d'une réconciliation et d'une « confraternité de l'humanité ». Difficile d'être plus clair! Ses positions politiques concernant la Belgique n'échappent pas non plus à ce message: "Nous démontons les cloisons étanches existant entre flamands et wallons, comme nous aidons à les démolir entre les autres peuples. » (Pansaers, III, 1918 : 119)

En l'absence d'un discours initial, le texte Poésie de René Verboom (1891-1955) ${ }^{14}$, l'auteur le plus présent dans la revue après Pansaers, peut cependant faire figure de déclaration 
d'intentions. Conçu comme une ode à la poésie comme seul moyen de transcender le réel, d'illuminer le présent et d'atteindre l'immortalité, le texte du poète montois est dédié à Pansaers, qui lui offre une de ses premières tribunes littéraires. En tant que forme d'expression libératrice, la poésie s'y trouve personnifiée en une femme « trop belle pour ne pas être surhumaine » (Verboom, I, 1917:19) qui agit, plus qu'en muse, en véritable démiurge. C'est par et grâce à l'écriture que l'homme retrouve la lumière de l'espoir et de la vie :

Impulsion étoilée, ô Poésie, tu m'as créé

du cœur à la pensée puisque je crée

à ton image souriante, infinie,

les figures suprêmes et lumineuses de la vie (Verboom, I, 1917 : 19)

11 La deuxième partie de ce premier poème entre en plein dans l'ode à la femme en tant que matérialisation corporelle. Cette approche sensuelle se reproduira dans Nocturnes (II, 1918: 59), alors que les autres poèmes - Pluie (I, $1917: 18$ ) Pochade (II, 1918: 60), Captivité (III, $1918:$ 83), Automne (III, $1918: 83$ ), Prière (IV, $1918: 154)$ et Les plus doux (IV, $1918: 155)$ - sont sans doute parmi les plus noirs de la revue, tous numéros confondus. Pochade évoque les espoirs brisés de la jeune génération :

Mais la nuit a broyé soudain le crépuscule

et l'ombre impérieuse qui dévore les choses

ronge le vase clair et absorbe les roses. (Verboom, II, $1917: 60$ )

Dans Captivité, dont le titre dévoile les tensions qui assaillent Verboom, le poète parle de "rêves fracturés» (Verboom, II, 1918: 83) mais aussi de l'espoir de rédemption par l'écriture : «Captif entre mes murs sans flamme et sans trophée, /J'hymnerai la Beauté tragique de la vie! » (Verboom, II, $1918: 83$ ) De son côté, Prière rassemble les rêves déçus et les peurs qui deviennent physiques tout en questionnant l'avenir avec angoisse : « Que vais-je donc adorer? Que vais-je donc souffrir? / Quelle chimère va mourir » (Verboom, IV, 1918 : 154) Dans ses vers encore marqués de l'esthétique symboliste, le poète imprime les questionnements d'une génération tiraillée entre l'envie de renouveau et l'angoisse de l'inconnu, avec l'écriture comme point d'horizon.

13 À l'instar de Verboom, Michel de Ghelderode a fait lui aussi ses premiers pas littéraires dans Résurrection. Dans le deuxième numéro, il publie La Légende du lierre (Ghelderode, II, 1918: 71-76) qu'il dédie " au poète Albert Lepage $»^{15}$, un conte encore maladroit où le futur auteur d'Escurial raconte une histoire d'amour brisée puis reformée par la mort, sur fond de mystique ingénue. Deux mois plus tard, il propose un autre conte, plus élaboré, Le baiser sur l'eau (Ghelderode, IV, 1918 : 147-154) à propos duquel il écrira : «Ces pages sont les premières que je reconnus en toute sincérité ${ }^{16}$. Dans ce conte aux airs naïfs, Ghelderode met en scène l'homme sincère et amoureux qui se voit éconduit par la femme légère et coquette : « Là, qui attend ses lèvres, le baiser repose sur l'aimabilité de l'eau. Le baiser, il sera rien... Et ses lèvres se tendent vers la fraîcheur - parfumée encore de la carnation affolante de Minnie. » (Ghelderode, IV, 1918 : 153).

Tout autre est le style imprimé aux deux poèmes de Raoul Ravache que contient la série. Le premier, intitulé de manière provocatrice Défi! se tourne tout entier vers l'avenir. Y domine le champ lexical de la force et de la puissance, valeurs faisant office de sésames vers le bonheur :

Heureux de ma puissance, heureux de ma clarté ${ }^{17}$,

Faisant bondir ma Vie ainsi qu'une lionne,

J'irai tout droit sans que mon âme se questionne

Lever au ciel les écussons de la Beauté. (Ravache, I, 1917 : 17) 
Moins combatif mais tout aussi déterminé à faire table rase du passé, le poème Janvier (Ravache, III, 1918 : 81-82) s'impose comme un cri lancé à la jeunesse, que le poète invite à « ensevelir/L'année défunte, la pauvre année » et à se diriger sans plus se retourner vers «l'avenir / [qui] Nous tend là-bas ses lèvres vierges. » Témoin de l'espoir de renouveau qui irrigue la nouvelle génération, Janvier utilise l'image de la blancheur de la neige pour la faire correspondre à la pureté que Ravache prétend retrouver après le désastre afin de construire un monde meilleur.

\section{Autour de l'expressionnisme allemand}

Les premier et second numéros consacrent une trentaine de pages à l'enquête réalisée par Clément Pansaers sous le titre "Autour de la littérature jeune-allemande ». Ouvrir le premier numéro de la revue, en 1917, par un essai sur la littérature jeune allemande ${ }^{18}$ et le dédier à Romain Rolland ne manque pas d'audace et indique entre autres une prise de position en faveur d'une réconciliation des peuples par le truchement de l'art, qui transcende les différences. Dans son analyse, Pansaers ne s'en tient pas uniquement à la production d'outre-Rhin mais brasse toute une série de noms et d'époques, souvent de manière décousue et sans véritable ligne méthodologique. Analyse (pseudo)historique à l'appui, l'auteur critique, entre autres, l'évolution fatale vers le pessimisme schopenhauérien et nieztschéen et l'apogée littéraire du spleen baudelairien. « Dès lors, la longue phalange des petits poètes miaulaient leurs strophes pleurnicheuses. Ce fut le poncif de la tristesse. Jusqu'avant la guerre la caste existait encore, de ceux qui n'ont jamais souffert, ou incidemment, mais qui saignaient volontiers et volontairement du cœur. L’Allemagne n'a pas fait exception. » (Pansaers, I, $1917: 16)^{19}$ En réaction, il en appelle au retour à la joie comme point d'orgue de cette revitalisation par la littérature : « Tout le monde crée de la tristesse. Il est plus facile à l'homme d'être triste que d'être gai. (...) L'humanité éprouve un besoin impérieux de se ressaisir dans la joie. C'est l'œuvre rédemptrice du poète de ce jour. » (Pansaers, II, $1918: 58$ ).

le concert de ses contemporains, on reconnaît les noms de ceux qu'il a pu lire dans les revues expressionnistes Die Aktion et Der Sturm ${ }^{20}$, et d'autres qui prendront aussi place dans Résurrection: Franz Werfel, Franz Blei, Carl Sternheim, Ernst Stadler, Frank Wedekind, entre autres. En Sternheim, Pansaers voit une sorte de produit syncrétique des sensibilités germaniques et latines (Pansaers, II, 1918 : 54) capable de percer la conscience humaine et de la libérer des atavismes. Pour Pansaers, Herwarth Walden a produit les œuvres constituant la " nouvelle étape de la littérature jeune allemande » (Pansaers, II, 1918 : 58) en lui apportant l'amour - sur l'absence duquel, dans les lettres germaniques, Pansaers disserte abondamment - et « ce qui a manqué à la littérature de tous les peuples européens, ce dernier quart de siècle - la joie. » (Pansaers, II, 1918 : 58) On voit bien ici comment le Pansaers d'avant Dada cherche à définir une esthétique à laquelle il pourrait se rattacher pleinement.

Pour Pansaers, « tout art est politique » (Pansaers, II, $1918: 53$ ) et il s'interroge :

Faut-il que l'humanité devienne de plus en plus un amalgame de tares et d'atavismes? Pourquoi ne pas se justifier devant soi-même, si inconsciemment on justifie déjà tellement le passé ? (...) Mais le passé toujours, est néfaste. Toute une vie est souvent insuffisante à détruire complètement en soi, ce passé, la vraie déchéance. Le monde occidental s'engourdit au passé. (Pansaers, I, 1917 : 15) 

avec la manière dont il parle de la guerre, presque par euphémisme - «la présente catastrophe » (Pansaers, I, $1917: 11$ ), « les événements de 1914 » (Pansaers, I, 1917 : 15), comme si, ne pas la nommer ouvertement, c'était déjà l'assumer comme un événement passé $^{21}$ et établir un rapport au monde entièrement renouvelé. Pansaers écrit :

Avant la guerre, le monde semblait ignorer la merveille qu'est la vie. Maintenant que l'humanité s'est avancée jusqu'au bord de la vie, qu'elle a escaladé les crimes, elle s'en rendra un meilleur compte. (...) Pourquoi, arrivés à un certain âge, nous croyons-nous parfaits, au lieu de continuer à naître dans la vie et ses merveilles. (Pansaers, II, 1918 : 52)

Un homme nouveau doit surgir des cendres du passé et imposer un tout autre rapport au monde : tel est le credo proclamé par Résurrection à chacune de ses pages et que les textes d'auteurs allemands que la revue publie - en (souvent mauvaise) traduction par Pansaers - s'appliquent à montrer. Ernst Stadler et son célèbre Départ (Der Aufbruch) est présent dans le premier numéro. Féroce dénonciation du militarisme prussien, l'extrait choisi clame avec force et violence l'absurdité d'une guerre qui décime sa jeunesse. Il est suivi par l'adresse Au lecteur de Franz Werfel, à forte teneur unanimiste : "Que tu sois nègre, acrobate, ou repose insouciant encore sous l'aile maternelle, (...) Que tu sois soldat, ou aviateur, plein d'endurance et de courage. » (Werfel, I, 1917 : 22) Par l'écriture, le poète communie avec les sentiments et le vécu de l'humanité toute entière. Le dernier vers lance un appel à la fraternité universelle : «Que, frère, nous nous tombions entre les bras, nous! » (Werfel, I, $1917: 22)$

21 Hommage politique, Jaurès accapare l'attention d'un autre représentant de l'expressionnisme allemand. Connu pour être l'auteur de Der Sohn (Le Fils, 1916), première pièce expressionniste jouée en Allemagne, Walter Hasenclever (1890-1940) s'engage d'abord à la guerre dont il perçoit rapidement l'inutilité. Devenu un fervent antimilitariste, il s'oppose plus tard au régime nazi et émigre en France où il se suicide lorsque l'Allemagne envahit la France en 1940. Construit en deux parties, le poème Jaurès place le socialiste français en position de messie sacrifié sur l'autel de la paix mais dont la mort est suivie d'une résurrection salvatrice pour l'humanité. Véritable appel à la concorde européenne laïque - «Soldats d'Europe! Les églises dévastées/ne sauvent jamais plus vos pays./Soldats d'Europe! Citoyens d'Europe !/écoutez la voix, qui vous appelle des frères » (Hasenclever, II, 1918: 16-17) - le poème de Hasenclever est un hymne à la réconciliation des peuples.

Résurrection s'attarde encore sur l'œuvre de Frank Wedekind (1864-1918), à qui Pansaers rend hommage dans le cinquième numéro, à l'occasion de sa mort. Il le considère comme le dépositaire d'une "expertise fameuse de l'humanité», qui "allume une fête poignante » (Pansaers, V, 1918: 193) ; la revue publie un extrait du célèbre Franziska (1912) dans cette même livraison, avec un choix significatif de deux extraits - traduits par Pansaers - qui dénoncent l'institution du mariage et la pression de la famille.

Quelques autres textes issus du corpus allemand convergent encore dans le sens d'une libération de l'humanité fourvoyée, comme celui d'Iwan Goll proclamant le nécessaire combat de l'art " contre l'inertie et le crétinisme » et contre « la nullité de la nuit » (Goll, IV, 1918: 121). Ou Alfred Wolfenstein (1883-1945) et son Bonheur de l'extériorisation (Wolfenstein, V, 1918 : 192-193) appelant à l'action et la parole des hommes contre le silence. Plusieurs extraits du célèbre Bébuquin (1913) de Carl Einstein apparaissent également dans les numéros IV et $\mathrm{V}$, dans une version française à nouveau traduite par 
Pansaers. Cuvre déroutante, parfois qualifié d'anti-roman ou d'a-roman, il s'agit dans tous les cas d'un texte qui a marqué son époque et auquel se sont référés plusieurs auteurs, en Allemagne et ailleurs. L'absence de linéarité au profit d'une multiplicité d'intrigues complémentaires ou, au contraire, disparates, un système d'énonciation indéfinissable, ainsi qu'une temporalité éclatée en font un texte résolument iconoclaste, dont la rédaction débute dès 1906. Outre les liens d'amitié qui lient Pansaers à Sternheim, on peut lire l'insertion de ces trois extraits de Bébuquin comme l'affirmation de l'inscription de la revue dans une avant-garde subversive et progressiste. Enfin, les passages cités n'ont sans doute pas été choisis au hasard et entrent en résonance avec les intentions de la revue:«(...) tout n'existait que dans l'anéantissement. Ici il vit un privilège de toute esthétique; mais aussitôt ne voyant plus de but final, il dut nier l'individuel. Il désira avidement la démence, mais son reste d'humain débridé en eut fort peur. » (Sternheim, IV, $1918:$ 156)

\section{D'autres ouvertures} allemande en français, c'est d'avoir mis celle-ci en relation avec le panorama lyrique français, dont l'unanimisme. Ainsi s'explique la présence de textes du premier Pierre-Jean Jouve, Jean Saint-Prix, Marcel Martinet et Charles Vildrac (1882-1971). De ce dernier, dont on se souviendra qu'il est à l'origine, avec Georges Duhamel, du Groupe de l'Abbaye (1906-1908), la revue publie le beau poème Les deux buveurs (Vildrac, I, 1917 :p. 23-24), clamant ce désir de fraternité au-dessus des rivalités. Véritable injonction à préférer le joie et l'entente cordiale plutôt que l'affrontement gratuit - « Ils pourraient se battre sans raison/Ils pourraient éveiller les mille raisons/Qu'ils ont de se battre» (Vildrac, I, 1917 : 24) - les vers de Vildrac témoignent aussi de l'effort de ces auteurs français pour creuser des voies neuves, permettant de concilier lyrisme et transformation sociale.

Tristesse (Jouve, I, 1917 : 32-33), Juste vent d'automne (Jouve, I, 1917 : 33-34), et Voix dans les temps terribles (Jouve, III, 1918 : 118) sont les titres des trois poèmes de Pierre-Jean Jouve (1887-1976) que publie Résurrection. Le dernier est un hymne à l'espoir humaniste, que le poète déclame en ayant recours à l'anaphore $d u$ "Je ne crains pas » qui scande le poème. Irradié par une lumière que l'on devine déjà divine, Jouve proclame sa foi envers l'homme ${ }^{22}$, au-delà et malgré la mort, et réclame de la douceur pour sa rédemption.

De Jean de Saint-Prix (1896-1919), la revue publie dans la seconde livraison, le poème Veillée. Ce pacifiste, membre après la guerre des Étudiants socialistes-révolutionnaires, a entretenu une correspondance d'un an et demi avec Romain Rolland. Convaincu de la propagation bénéfique sur toute l'Europe des vents nouveaux venus de l'Est, il publie dans Résurrection, des vers brefs dédiés à la Révolution russe. Lors de son exil suisse de la guerre, où il rencontre Romain Rolland, il fait également la connaissance de Marcel Martinet (1887-1944), qui militera comme lui pour l'adhésion à la Troisième Internationale. Avant et pendant la guerre, à laquelle il ne participe pas pour raisons de santé, ses positions de gauche antimilitaristes sont connues. C'est de fait un poème foncièrement antimilitariste, Tu vas te battre, qui est publié dans le troisième numéro de la revue. Martinet y dénonce avec véhémence l'absurdité de la guerre qui oppose des travailleurs autrefois unis dans une même lutte politique :

Constructeurs de cité, âmes libres et fières,

Cœurs francs, vous étiez là, frères d'armes, debout. 


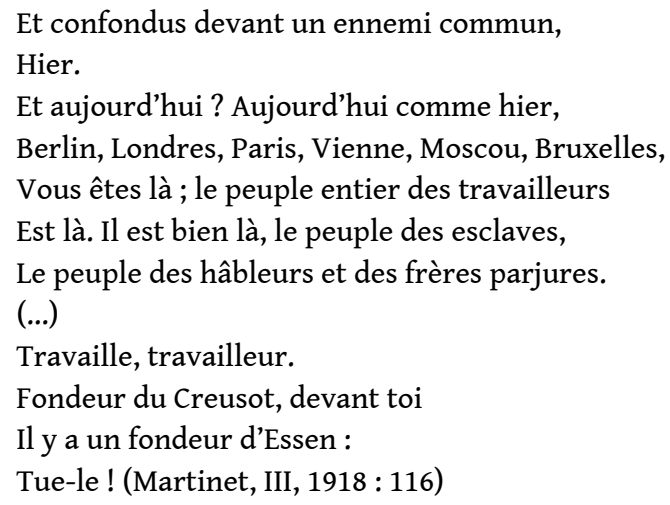

\section{Pansaers écrivain}

Outre son rôle d'animateur et de directeur, Clément Pansaers est l'un des plus importants contributeurs de la revue. J'ai déjà parlé de son long commentaire à la Littérature jeune allemande, qui s'étend sur les deux premiers numéros, ainsi que de l'hommage qu'il rend à Frank Wedekind (Pansaers, V, 1918: 193) mais d'autres textes critiques trouvent également place dans la revue. Dans la troisième livraison, Épilogue compte près de huit pages et est tout entier innervé du questionnement sur le sens de la volonté humaine - au sens collectif et individuel - et sur les dérives, voire les déviances de la "civilisation contemporaine » que Pansaers assimile à une «bielle, [un engrenage]» (Pansaers, III, 1918 : 110). Texte pamphlétaire aux allures dadaïstes, il dénonce aussi la marchandisation de l'art et l'opportunisme de certains - "Le pivot de la rente secondaire dans la civilisation contemporaine est le verbe réfléchi - - se faufiler. (...) Cependant que le faufileur tient toujours un doigt au-dessus de la tête afin de sentir d'où vient le vent " (1918: 111) - et souligne l'extrême difficulté de rester soi-même, de défendre des valeurs auxquelles on croit, indépendamment de la classe sociale - "aristocrate, bourgeois, prolétaire »- de son interlocuteur.

Dans une langue beaucoup plus classique, Pansaers envisage le rôle de l'artiste dans le dernier numéro, avec la réflexion intitulée Brève incursion dans le Blockhaus de l'artiste. Pansaers y réclame des œuvres d'art qui aident l'humanité plutôt que celles qui célèbrent l'artiste lui-même :

Le poème a d'autant plus de valeur qu'il aide un plus grand nombre d'humains à vivre ! Est intéressant, jusqu'au génie, celui qui sait donner une forme sensible à ce que le monde entier, homme, pierre, arbre, etc. pensent et sentent et qui jusqu'ici, fut inexprimé encore. (Pansaers, V, $1918: 204$ )

Or, précise-t-il, il récuse la fonction «spécifiquement social[e] »(Pansaers, V, 1918 : 208) de l'art. Le caractère premier de l'art et son authenticité entrent pour Pansaers en forte contradiction avec le carriérisme ou la quête de reconnaissance que poursuivent d'aucuns ou l'opération de mythologie à laquelle on les soumet: "A travers l'œuvre, l'humain cherche son semblable (c'est bien) mais d'une façon mythique (c'est le monde à l'envers). Pensez au biographe, au conférencier qui, toujours pour se rendre lui-même intéressant, alambique le mythe. » (Pansaers, V, 1918 : 206)

D'un point de vue plus strictement littéraire, Pansaers propose aux lecteurs de Résurrection des textes de nature diverse. Les longs poèmes Méditations de Carême: L'Interrègne (Pansaers, I, $1918: 25)$ et le tendre Novénaire de l'Attente ${ }^{23}$, dédié « À toi, fils, ce cantique de tes neuf lunes pleines. 1916-1917» (Pansaers, IV, 1918: 136) dévoilent les 
influences de la philosophie taoïste avec laquelle Pansaers se familiarise dès 1916 (Dachy, 1973). Comme dans son Point d'orgue programmatique pour un jeune orang-outang, l'auteur y fait appel aux forces cosmiques et aux valeurs alchimiques de l'univers comme modes de sublimer le réel. Il clame un besoin de rénovation et une recherche d'authenticité, tout en apercevant déjà les signes de ce changement :

Le monde est en-dessous de la flottaison.

La terre, lasse, a besoin de se modifier.

Obstruée de faux - semblants

et d'autres parrainages gratuits -

déjà la voirie surgit désencombrée, commode. (Pansaers, I, 1918 : 26)

31 La nature et ses forces demeurent comme un cadre formel, elles guident le cours des événements et leur donnent la force d'aborder l'étape de modification qui engloberait l'humanité toute entière.

Appel à la fraternité universelle, Novénaire de l'attente se veut aussi un chant à la vie contre l'absurdité de la haine et le triomphe du mensonge :

Venez - imposteurs de la vérité !

Que je vous montre le sacrifice sublime de votre être... La haine est une vertu?

(...)

Frères !

Haïssez - en grands vertueux -

les vices de vos subconsciences

et ceux de vos viscères -

Mais - aimez follement vos frères

Comme le soleil adore la Terre ! (Pansaers, IV, 1918 : 138)

Témoignage de Pansaers à son fils à naître, les vers qu'il lui consacre montrent à la fois la générosité et l'angoisse légitime du futur père :

Le jour de ta naissance

je presserais [sic] mes lèvres sur ton nombril

- le stigmate du néant.

Tu n'as pas encore une âme

- Inanimé -

Veux-tu que je te passe la mienne?

Je sais qu'elle est pareille

à une absurdité crasseuse

usée comme une sandale

au pied d'un moine saltimbanque

(...) qui erre de Jérusalem à la Mecque.

Mais tu monterais ton existence

sur ma triste expérience

(...)

Orgueilleux et hautain

promène ton identité avec prestance -

comme un poisson dans l'eau

le cygne sur l'étang. (Pansaers, VI, 1918 : 222-224)

Enfin, il faut comprendre Les Saltimbanques, emblématiquement sous-titré Comédie du Polyèdre pour marionnettes vivantes (Pansaers, V, 1918 : 165-171), la seule création théâtrale de Pansaers publiée dans Résurrection, comme une sorte de mise en abyme du genre théâtral ${ }^{24}$. En effet, le théâtre y apparaît comme un "système pris à son propre jeu, le dialogue y servant une forme d'humour absurde et babélien" (Dachy, 1973) où les 
personnages «ne semblent doués de parole que pour mettre en évidence l'incommunication qui les sépare. " S'y succèdent aphorismes, proverbes et sentences sans lien apparent les uns avec les autres et où l'idée de l'incommunicabilité entre les hommes et la vacuité du langage se trouvent soulignées à diverses reprises :

L'AGRÉGÉ ÈS-FOLIES - Inoffensif, je m'adresse aux arbres, aux maillots et à tout ce qui ne parle pas encore.

LE POLYÈDRE - Discussion inutile. Une idée travestie en paroles est perdue pour l'humanité.

L'ILLUMiNÉ - Réformes! L'humanité est infecte. Désinfecter, bénigniser.

Imbéciles! (Pansaers, V, $1918: 168)$

Par ailleurs, cette dernière réplique renferme l'une des idées majeures qui parcourt les six numéros, à savoir que l'humanité doit se donner pour mission, en 1917-18, de se libérer de tout ce qui est nocif pour elle et de procéder à un bouleversement, qui, outre l'art et la littérature, doit aussi passer par l'engagement politique. Clément Pansaers utilise en effet Résurrection comme une tribune ${ }^{25}$. Ainsi les bulletins situés à la fin de chacun des six numéros constituent-ils autant d'occasion de diffuser ses opinions, qu'elles soient plus générales ou concernent spécifiquement la Belgique ou la cause prolétarienne.

\section{Conclusion}

Que retenir de la brève existence de Résurrection? D'une part, on se souviendra que la revue a fait connaître des auteurs expressionnistes allemands majeurs, dont on ignorait à peu près tout dans l'espace francophone. Ensuite, elle a recueilli les premières manifestations avant-gardistes, dont la modernité contraste avec le ton patriotique des poètes des tranchées, notamment ceux réunis, à la même époque, dans Les Cahiers, fondés au front pour la Défense et l'Illustration de la Langue Française en Belgique. D'autre part, la revue a offert l'occasion à de jeunes auteurs belges d'être publiés pour la première fois, comme René Verboom et Michel de Ghelderode. Enfin, dans la sphère belge, il convient de souligner l'apport de Résurrection au pacifisme, tel qu'on le célébrait alors en France et en Allemagne et qui était pratiquement absent du paysage littéraire et intellectuel belge. À cette cause, Pansaers ajoute une admiration de la révolution russe et, inscription locale oblige, ses prises de position fédéralistes.

Point de ralliement de l'avant-garde qui annonce le surréalisme, œuvre essentielle d'un seul homme - Clément Pansaers - Résurrection ne s'inscrit pas seulement dans l'histoire littéraire en tant que point de repère mais aussi «comme le lieu de nouveaux départs possibles.» (Dachy, 1972) La variété des contributions n'a d'égale que la volonté d'atteindre un pacifisme universel à travers la littérature mais aussi grâce à l'engagement moral, voire politique, des artistes qui les produisent. Résurrection porte aussi la marque d'une inscription historique forte, soumise aux tensions d'une époque tumultueuse et complexe, qu'elle tente de sublimer par la pensée et la création afin que, sur les ruines d'un passé qu'elle veut s'empresser d'oublier, une nouvelle génération retrouve la concorde et la fraternité universelle, sur fond du communisme qui offre encore de beaux espoirs. 


\section{BIBLIOGRAPHIE}

BARONIAN, Jean-Baptiste (2003). « Pol Demade, un petit maître belge du fantastique » [en ligne]. Bruxelles : Académie royale de langue et de littérature françaises de Belgique. [disponible le 13 avril 2015] URL : http://www.arllfb.be/ebibliotheque/communications/baronian130903.pdf. DACHY, Marc (1973) «Résurrection de Clément Pansaers », in Résurrection. Cahiers mensuels littéraires illustrés. Collection complète. 1917-1918, Bruxelles : Éditions Jacques Antoine, s.p. Dumoulin, Michel (2006). «L'entrée dans le xx ${ }^{\mathrm{e}}$ siècle », in Michel Dumoulin, Vincent Dujardin, Emmanuel Gerard, Mark Van den Wijngaert (dir.). Nouvelle Histoire de Belgique. Bruxelles : Éditions Complexe, pp. 1-167.

KoENIG, Théodore (1971). « Dada », in Phantomas. Dossier La Belgique sauvage. N 100-111, déc. 1971, p. 194-196.

DE MARNEFFE, Daphné (2005). « Assassiner Dada : Pansaers et le numéro spécial de Ça ira! (1921) », in Henri Béhar, Catherine Dufour (dir.) Dada. Circuit total. Lausanne : L'Âge d'Homme. Coll. « Les dossiers H », pp. 324-332.

PANSAERS, Clément (1972). Sur un aveugle mur blanc et autres textes. Lettres à Tzara, Picabia et Van Esche. Édition établie par Marc Dachy, Bruxelles :TRANSédITION.

QUAGHEBEUR, Marc (1998). Balises pour l'histoire des lettres belges [première édition 1982]. Bruxelles : Labor. Coll. « Espace Nord».

Résurrection. Cahiers mensuels littéraires illustrés. Collection complète. 1917-1918 (1973). Bruxelles : Éditions Jacques Antoine.

ROLAND, Hubert (1994). « Résurrection, Clément Pansaers et Carl Sternheim : "comment rendre une revue intéressante" ", in Les Lettres romanes, tome XLVIII, n³-4, p. 259-275.

ROLAND, Hubert (2003). La «Colonie » littéraire allemande en Belgique. 1914-1918. Bruxelles : AML Éditions/Éditions Labor. Coll. « Archives du Futur ».

SAUWEN, Rik (1969). L'esprit Dada en Belgique. Mémoire présenté pour l'obtention du grade de licencié en philologie romane, Louvain : Katholieke Universiteit Leuven.

DE SCHAEPDRIJVER, Sophie (2004). La Belgique et la Première Guerre mondiale. Bruxelles : PIE Peter Lang.

WEISGEBER, Jean (2001). « Un pays ouvert aux quatre vents », in Revue de littérature comparée, $\mathrm{n}^{\circ}$ $299,3 / 2001$, p. 353-361.

WILLINGER, David (2002) Theatrical Gestures of Belgian Modernism. Dada, Surrealism, Futurism and Pure Plastic in Twentieth-Century Belgian Theatre. New York : Peter Lang 


\section{NOTES}

1. Le mémoire présenté par Rik Sauwen en 1969 dévoile pour la première fois, quelque cinquante ans plus tard, l'aventure de Résurrection, de Pansaers et du mouvement Dada en Belgique, complètement négligée jusque là par l'historiographie littéraire. Ainsi, Clément Pansaers n'apparaît pas dans la somme pourtant riche de Camille Hanlet, Les Écrivains belges contemporains de langue française, 1800-1946, publiée en deux tomes en 1946, aux liégeoises éditions Dessain.

2. Même si son existence fut éphémère : «Résurrection ne fit pas honneur à son nom ; elle n'eut rien d'éternel, pas même un lendemain. » (Weisgeber, $2001: 359$ )

3. Voir l'article de Marc Quaghebeur dans ce même numéro, "Jeunes écrivains durant la première occupation allemande de la Belgique ".

4. Dans son Tagebuch, Thea Sternheim, pourtant d'abord méfiante envers cet inconnu que son mari Carl lui impose comme précepteur de leurs enfants, est catégorique. Pour elle, aucun doute ne plane sur les convictions de Pansaers: «Les renseignements sur son compte parlent de "Flamingand". Il n'en est rien du tout. Je ne peux pas juger des qualités de cet homme mais je suis de prime abord convaincue de la sincérité de ses opinions pacifistes. Il est au-dessus de la guerre, ce qui signifie déjà énormément de nos jours. » (cité et traduit par Hubert Roland, 1994 : 263)

5. Hubert Roland a examiné des documents d'époque, issus des archives militaires du Auswärtiges Amt (Ministère allemand des affaires étrangères). «Ils illustrent la curieuse concordance d'intérêts entre l'idéalisme internationaliste de Pansaers et les considérations stratégiques de l'administration allemande à travers une même thèse : celle d'une Belgique fédérale comprenant deux entités autonomes. » (Roland, $1994: 261$ )

6. Voir plus particulièrement le chapitre consacré à l'aventure de Résurrection, sous le titre «Ententes littéraires belgo-allemandes» (Roland, 2003: 171-192), ainsi que son article « Résurrection, Clément Pansaers et Carl Sternheim (...) » (1994 : 259-275)

7. Une lettre datée du 30 octobre 1918 le situe déjà à Berlin, d'où il tente de se rendre en Suisse. Adressée au sous-secrétaire d'État Eduard David au Ministère des Affaires étrangères, c'est un Pansaers sans le sou qui demande à la fois un passeport et un peu d'argent pour financer son voyage. Il réitère sa demande avec insistance le 2 novembre 1918, en demandant en outre de quoi payer sa chambre d'hôtel et en annonçant sa décision de regagner finalement Bruxelles, ce qu'il finira par faire (Roland, 2003 : 186-187).

8. Sur les traces postérieures de Dada en Belgique, voir Koenig, 1971.

9. Sur le parcours dadaïste de Pansaers, voir de Marneffe, 2005.

10. Pour Hubert Roland, "le financement de Résurrection fut garanti par une "connection" allemande très probablement établie par Sternheim [...]» (Roland, 1994 : 260).

11. Toutes les références à la revue dans cet article sont indiquées de la sorte : nom de l'auteur, numéro de la revue, année : page, celle-ci correspondant à la pagination de la réédition de 1972.

12. Von der Lacken, Tätigkeitsberichte, Anfang August 1917-Ende Januar 1918, p. 30 (voir Roland, 1994 : 269-273 et Roland, $2003: 184)$.

13. Marc Quaghebeur (1998) avait inclus Pansaers, dès la première édition de ses Balises en 1982, dans le chapitre «Du tourbillon des avant-gardes émergent trois figures d'une radicalité absolue ", à côté de Paul Nougé et Henri Michaux.

14. Dont la production lyrique a été rassemblée en 2004 sous le titre de La courbe ardente (tome I : 1915-1945 et tome II : 1946-1949), par les éditions de l'Arbre à Paroles.

15. Le chassé-croisé de dédicaces dans les divers numéros de la revue mériterait à lui seul une analyse poussée. Ainsi les hommages et marques d'allégeance ne manquent-ils pas. Dans un curieux jeu de renvois en miroirs, Raoul Ravache dédie son Janvier à Paul Windfohr, qui consacre à son tour sa Force à René Verboom qui avait dédicacé son Poésie à Clément Pansaers. 
16. Voir les notes manuscrites de l'auteur sur le tiré-à-part de la revue (Document conservé aux AML sous la cote MLT 05249/0018).

17. Soulignons que Clarté sera aussi le nom donné à la revue animée par Henri Barbusse de 1919 à 1921.

18. Dans son journal, en date du 31 mars 1917, Théa Sternheim évoque les recherches de Pansaers sur cette littérature, qu'elle considère trop peu rigoureuses et trop peu étayées par des lectures : «Comment en outre Pensaers [sic] peut-il juger Blei, Werfel et de tels gens, dont il n'a pas lu dix lignes. [...] [Son essai] est malheureusement la somme de quelques conversations avec Karl [Sternheim], pour la plupart mal comprises, à peine révisées par une lecture distraite et qui se présente d'une façon confuse.» (Hubert Roland, 2003 : 176-177). Si je ne puis personnellement pas préjuger des divergences éventuelles entre ce que Théa Sternheim entendit et ce que Pansaers publia dans Résurrection, il n'en demeure pas moins que le texte édité est en effet, à bien des égards, confus et décousu.

19. Pour Sauwen (1969: 64), ce type de considérations marque clairement la parenté, avant même de l'avoir formalisée, entre Pansaers et les théories dadaïstes de Tzara. En effet, la rencontre entre les deux hommes date de 1920 même si Pansaers découvre l'homme avant. Mais, en tous les cas, lorsqu'il écrit ces lignes, le manifeste dada de 1918 n'a pas encore été écrit, dans lequel on peut lire «Nous avons bousculé le penchant pleurnichard en nous. Toute filtration de cette nature est diarrhée confite. » (cité par Sauwen).

20. Et qui sont aussi ceux pour qui Sternheim montre le plus de considérations (Roland, 1994 : 265).

21. On peut légitimement s'interroger sur ses convictions en 1917 : croit-il encore à la victoire allemande?

22. Le numéro III de la revue annonce la participation du Français à une lecture publique de ses œuvres, organisée conjointement avec celles de Stefan Zweig, à Zurich, « en une manifestation confraternelle et artistique. » (p. 120)

23. Fourni en trois livraisons : IV, p. 136 ; V, p. 181 ; VI, p. 219.

24. Le texte est publié en version anglaise dans l'anthologie commentée par David Willinger (2002) Theatrical Gestures of Belgian Modernism. Dada, Surrealism, Futurism and Pure Plastic in Twentieth-Century Belgian Theatre. New York : Peter Lang.

25. Ce volet est plus largement développé dans l'article de Marc Quaghebeur de ce même numéro.

\section{RÉSUMÉS}

En tant que revue belge d'avant-garde publiée pendant la Première Guerre mondiale, Résurrection (1917-1918) rend compte des doutes mais aussi de l'espoir qui dominent cette période complexe. Animé par Clément Pansaers, le périodique rassemble des contributions d'auteurs belges, français et allemands, dans une volonté manifeste de sublimer le réel par l'imaginaire et de puiser dans la littérature les ferments d'un monde nouveau, dont les auteurs pressentent l'avènement. Une analyse du contenu des 6 numéros dévoile son caractère hétéroclite, avec une dominante de textes expressionnistes mais aussi (pré)dadaïstes et unanimistes. L'article se penche aussi sur la ligne politique de la revue, que marque son directeur. Y dominent par-dessus 
tout la recherche du maintien de l'humanisme malgré la barbarie de la guerre et un appel fervent à la concorde des peuples.

An avant-garde Belgian review published during World War I, Résurrection (1917-1918) reveals both the doubts and the hopes that dominate this complex period in history. Edited by Clément Pansaers, this periodical assembles contributions from Belgian, French, and German authors in an effort to enhance the real with the imaginary and to inspire a new world through literature. An analysis of six issues of Résurrection reveals its varied nature: expressionist texts dominate the publication, yet examples of predadaist and unanimist texts are featured as well. The article also focuses on the review's political aspect, a mark of the Pansaers' influence. Above all, the review emphasizes the necessity of upholding humanism in the face of barbarity and war, fervently calling harmony between people.

INDEX

Mots-clés : littérature belge, Première Guerre mondiale, revues littéraires, avant-garde, expressionisme

Keywords : Belgian literature, World War I, literary reviews, avant-garde, expressionism

\section{AUTEUR}

\section{LAURENCE BOUDART}

Archives \& Musée de la Littérature (Bruxelles)

laurence.boudart@aml-cfwb.be 